\title{
ACTIVIDAD ANTIOXIDANTE DE Tropaeolum tuberosum RUIZ \& PAVÓN (MASHUA) Y SU APLICACIÓN COMO COLORANTE PARA YOGUR
}

\author{
Antioxidant activity Tropaeolum tuberosum Ruiz \& Pavón (mashua) and its application as \\ colorant for yogurt
}

Luis A. Inostroza ${ }^{1}$, Américo J. Castro ${ }^{1}$, Eloisa M. Hernández ${ }^{1}$ Mario Carhuapoma ${ }^{1}$, Ricardo A. Yuli ${ }^{1}$, Amadeo Collado ${ }^{1}$, Javier S. Córdova ${ }^{2}$ ${ }^{1}$ Instituto de Investigación en Ciencias Farmacéuticas y Recursos Naturales "Juan de Dios Guevara”, Facultad de Farmacia y Bioquímica, Universidad Nacional Mayor de San Marcos. ${ }^{2}$ Centro Latinoamericano de Enseñanza e Investigación en Bacteriología Alimentaria - CLEIBA

\section{RESUMEN}

El objetivo del estudio fue evaluar la actividad antioxidante y uso del extracto etanólico de Tropaeolum tuberosum Ruiz \& Pavón (mashua) como colorante para yogur. La actividad antioxidante se determinó por los métodos DPPH y ABTS. El contenido total de antocianinas (AT) y polifenoles (PT) fue determinado por el método de $\mathrm{pH}$ diferencial y Folin-Ciocalteu, respectivamente. Los parámetros del sistema CIEL*a* ${ }^{*}$ se utilizaron para medir el color morado y la concentración de pigmento empleado para colorear el yogur natural "3500", comparándose con yogur comercial de mora "6224", que fue tomado como referencia. Ambas muestras de yogur se almacenaron bajo condiciones de refrigeración $\left(4^{\circ} \mathrm{C} \pm 1\right)$ durante 28 días; se les midió color y pH cada 4 días. Para el análisis sensorial se aplicó la prueba de aceptación $(p<0,05)$. El contenido de AT y PT en los extractos analizados fueron 192,63 \pm 1,28 mg de cianidina-3-glucósido/10og peso fresco (PF) y de 314,12 $\pm 1,23 \mathrm{mg}$ de ácido gálico/10og PF, respectivamente. La capacidad antioxidante equivalente al trolox obtenida fue de 15,8 $\pm 0,2$ $\mu \mathrm{mol} / \mathrm{g}$ PF, aplicando el ensayo DPPH, y 17,0 \pm o,2 $\mu \mathrm{mol} / \mathrm{g}$ PF con ABTS. El extracto se adicionó al yogur natural (1,2 mg/100 g) impartiendo un color morado similar al del yogur comercial de mora con mínimos cambios del color que no fueron visualmente evidentes; el yogur "350o" fue significativamente el preferido por los jueces frente al yogur "6224". Se concluye que los pigmentos extraídos del tubérculo de Tropaeolum tuberosum Ruiz \& Pavón, poseen actividad antioxidante, presentándose como una alternativa de colorante aplicable en alimentos de acidez intermedia como el yogur.

Palabras clave: Tropaeolum tuberosum, actividad antioxidante, antocianinas, polifenoles totales, colorante, yogur.

\section{SUMMARY}

The objective of the study was to evaluate the antioxidant activity and use of ethanol extract of Tropaeolum tuberosum Ruiz \& Pavón (mashua) as colorant for yogurt. The antioxidant activity was evaluated using DPPH and ABTS methods. The total content of anthocyanins (TA) and polyphenols (TP) was determined by the method of differential $\mathrm{pH}$ and Folin-Ciocalteu, respectively. Parameters of CIEL*a* $\mathrm{b}^{*}$ system were used to measure the purple color and concentration of pigment used to color natural yogurt "350o", comparing with yogurt commercial blackberry "6224" which was taken as a reference. Both yogurt samples were stored under refrigeration $\left(4^{\circ} \mathrm{C} \pm 1\right)$ for 28 days and color and $\mathrm{pH}$ were measured every 4 days. For sensory analysis the acceptance test was applied $(p<0,05)$. The contents of TA and TP in the extracts analyzed were 192,63 $\pm 1,28 \mathrm{mg}$ of cyanidin-3-glucoside/10og fresh weight (FW) and 314,12 $\pm 1,23 \mathrm{mg}$ of gallic acid/100 g FW, respectively. The antioxidant capacity trolox equivalent obtained was $15,8 \pm 0,2 \mu \mathrm{mol} / \mathrm{g} \mathrm{FW}$, using the DPPH assay, and $17,0 \pm 0,2 \mu \mathrm{mol} / \mathrm{g}$ FW with ABTS, the extract was added to plain yogurt $(1,2 \mathrm{mg} / 100 \mathrm{~g})$ imparting the purple similar to commercial yogurt blackberry color with minimal changes that were not visually apparent; yogurt "3500" was significantly preferred by judges against the yogurt "6224". It is concluded that the extracted pigments tuber Tropaeolum tuberosum Ruiz \& Pavon, possess antioxidant activity, appearing as an alternative of colorant aplicable intermediate acidity foods like yogurt.

Keywords: Tropaeolum tuberosum, antioxidant activity, anthocyanins, polyphenols, colorant, yogurt.

\section{INTRODUCCIÓN}

a primera sensación de un alimento que percibe el consumidor es su color, el cual

incluso influye en la aceptación o rechazo del mismo, de modo que los alimentos deben colorearse de manera artificial para hacerlos más atractivos. Con este fin pueden utilizarse colorantes obtenidos de fuentes naturales o mediante tratamientos físicos y químicos, sin embargo, no todas las sustancias colorantes son adecuadas para su uso en alimentos, ya que pueden resultar nocivas para la salud ${ }^{(1)}$.

La preocupación de los consumidores acerca de la seguridad de los colorantes artificiales ha incrementado la preferencia por el uso de colorantes naturales como aditivos ${ }^{(2)}$. Las restricciones en el uso de colorantes sintéticos en alimentos ha conducido al interés en el uso potencial de antocianinas como colorantes alimenticios en bebidas, jarabes, jugos de frutas, gelatinas, mermeladas, helados, dulces de pasta y yogures. También, en pasta dental, productos farmacéuticos, cosméticos y productos similares ${ }^{(3)}$.

Las recomendaciones dietarias en los últimos años proponen el incremento del consumo de alimentos que contienen fitoquímicos (carotenoides, flavonoides, índoles, isocianatos), ya que éstos proveen efectos beneficiosos para la salud humana y juegan un papel importante en la prevención de enfermedades crónicas (4). Las antocianinas son el grupo más importante de compuestos hidrosolubles, responsables de los colores rojo, azul y púrpura que se aprecian en flores, frutos y otras partes de las plantas. Su incorporación en alimentos tiene la ventaja no sólo de impartir color, sino que por las propiedades antioxidantes de las antocianinas, se pueden 
considerar como alimentos funcionales. Las antocianinas han sido consumidas por muchos años sin efectos adversos aparentes, tienen colores atractivos brillantes, y son solubles en agua, facilitando su incorporación en sistemas acuosos alimenticios (5). Los antioxidantes, incluyendo las antocianinas, interactúan con las especies reactivas del oxígeno, pudiendo mitigar sus efectos dañinos, por lo que jugarían un papel significativo en la prevención de las enfermedades ${ }^{(4)}$. Estas cualidades las hacen alternativas atractivas para su uso como colorantes naturales no sintéticos ${ }^{(6)}$.

Es cierto que no se puede calificar de malo a lo que a la vez se denomina alimento, pero los avances científicos en el ámbito de la salud ponen de manifiesto que la inclusión o exclusión de ciertos alimentos o ingredientes sí pueden conformar una dieta más o menos saludable. Esto, que para algunos puede llegar a considerarse de obsesión por la salud, ha dado lugar a que la industria alimentaria saque al mercado una serie de productos que satisfagan esta demanda e incluso, crearla cuando todavía no existe ${ }^{(7)}$. Debido al aumento en la población mundial, es importante la prevención y tratamiento de enfermedades y maximizar la calidad de vida. Dentro de los alimentos funcionales o alimentos que promueven la salud de las personas se encuentra el yogur, un alimento que ha crecido en popularidad año tras año debido, entre otros aspectos, a los beneficios percibidos en la salud ${ }^{(8)}$.

Tropaeolum tuberosum Ruiz \& Pavón (mashua), es una planta herbácea perenne, posiblemente originaria de los Andes centrales (10-20 ${ }^{\circ}$ lat. S); su cultivo se habría extendido por migraciones del hombre precolombino hasta Colombia $\left(8^{\circ}\right.$ lat. $\left.\mathrm{N}\right)$, el norte de argentina $\mathrm{y}$ Chile $\left(25^{\circ}\right.$ lat. S) ${ }^{(9)}$. Las mayores áreas de siembra se encuentran en Perú y Bolivia, entre 2400 a $4300 \mathrm{~m}$ de altitud, donde generalmente se cultiva en asociación con otros tubérculos, como la oca (Oxalis tuberosa), el ulluco (Ullucus tuberosus) y las papas (Solanum tuberosum) (10). El color de la piel de los tubérculos de Tropaeolum tuberosum R. \& P. pueden variar del blanco marfil al púrpura morado muy oscuro, pasando por el amarillo, naranja y púrpura morado en distintas tonalidades ${ }^{(9)}$; en nuestro país son subutilizados y con escasa demanda por los consumidores, aun teniendo elevado valor nutritivo con aporte de compuestos fenólicos, antocianinas, proteínas, carbohidratos, ácido ascórbico y otros principios bioactivos ${ }^{(10-12)}$.

A pesar de su importancia económica y del "boom" actual del "retorno a lo natural", la industria de los colorantes naturales no ha alcanzado aún, en nuestro país, el desarrollo que debía esperarse de un país poseedor de una inmensa biodiversidad. Nos propusimos ejecutar el presente trabajo de investigación con el fin de promover el mejor aprovechamiento de nuestros recursos naturales $y$, en estos, la búsqueda de nuevos colorantes naturales con capacidad antioxidante como son los tubérculos de Tropaeolum tuberosum Ruiz \& Pavón (mashua); de piel y pulpa morada. Su extracto etanólico aplicado al yogur natural permite elaborar un alimento funcional de color morado con alta actividad antioxidante; convirtiéndose en una alternativa sana y natural para quienes están dejando de lado los alimentos con colorantes sintéticos y para aquellos consumidores que cada vez están más conscientes de su autocuidado y buscan en el mercado aquellos productos que contribuyan a su salud y bienestar. Es de resaltar, además, que su valor nutricional lo hace muy recomendable para todos.

Por lo expuesto, el estudio tuvo como objetivo evaluar la actividad antioxidante y uso potencial del extracto etanólico de Tropaeolum tuberosum Ruiz \& Pavón (mashua) como colorante para yogur.

\section{MATERIAL Y MÉTODOS}

El diseño del estudio es de tipo experimental, longitudinal y prospectivo. Se realizó en el Instituto de Investigación en Ciencias Farmacéuticas y Recursos Naturales "Juan de Dios Guevara" de la Facultad de Farmacia y Bioquímica de la Universidad Nacional Mayor de San Marcos, en el Laboratorio de Biotecnología de la Escuela de Ingeniería Agroindustrial de la Facultad de Ciencias Agropecuarias de la Universidad Nacional de Trujillo y en el Laboratorio de Físico Química del Departamento de Criminalística de la Dirección de Investigación Criminal de la Policía Nacional del Perú.

\section{Material vegetal}

Los tubérculos de Tropaeolum tuberosum Ruiz \& Pavón (mashua), fueron colectados en el distrito de Pazos (3840 m), provincia de Tayacaja (figura 1), región de Huancavelica, y su clasificación taxonómica la realizó el Museo de Historia Natural de la Universidad Nacional Mayor de San Marcos, según el sistema de clasificación de A. Cronquist (1981):

Reino

División

Clase

Sub Clase

Orden

Familia

Género

Especie

: Plantae

Nombres comunes : Mashua (Perú, Ecuador), isaño, añu

: Magnoliophyta

: Magnoliopsida

: Rosidae

: Geraniales

: Tropaeolaceae

: Tropaeolum

: Tropaeolum tuberosum R. \& P.

(Perú, Bolivia), maswallo, mazuko, mascho (Perú), cubio (Colombia) ${ }^{(9)}$.

\section{Obtención del extracto}

Los tubérculos (4 kg) fueron triturados y homogeneizados con una solución de extracción de ácido clorhídrico $1,5 \mathrm{~N}$ y etanol al $96 \%(15: 85, \mathrm{v} / \mathrm{v})$, siendo la proporción de sólido a disolvente de (1:2, p/v). La mezcla obtenida se puso en maceración por 72 horas a $4^{\circ} \mathrm{C}$, en un frasco de color ámbar, con agitación diaria, posterior filtración y concentración en un evaporador rotatorio a $40^{\circ} \mathrm{C}$. Obtenido el extracto se colocó en tubos ámbar y se almacenó a $-18^{\circ} \mathrm{C}$ en atmósfera de nitrógeno hasta su análisis o aplicación al yogur ${ }^{(13,14)}$.

\section{Estudio fitoquímico}

Para determinar la presencia de metabolitos secundarios se realizaron diferentes ensayos con reactivos químicos de identificación, mediante cambios 


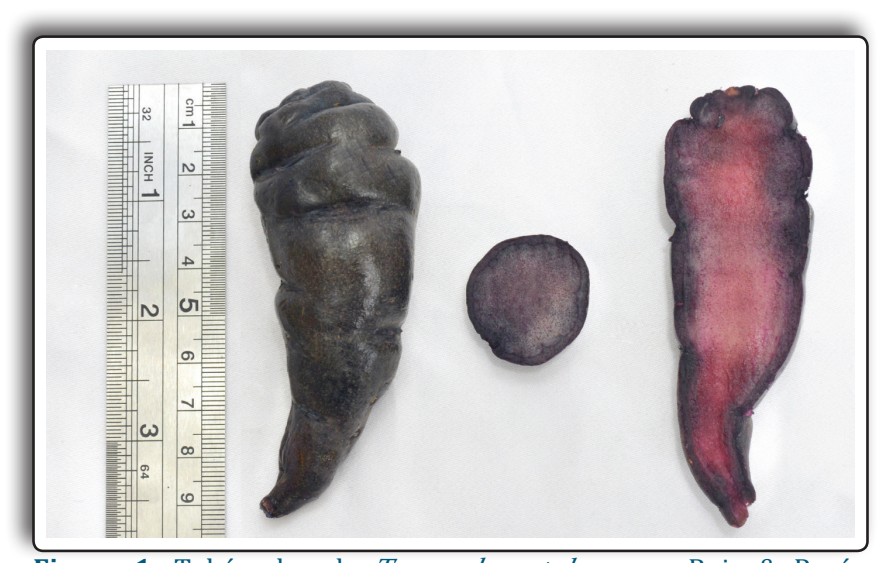

Figura 1. Tubérculos de Tropaeolum tuberosum Ruiz \& Pavón (mashua) de piel y pulpa morada.

de color o formación de precipitados, considerando: flavonoides (Shinoda), compuestos fenólicos (cloruro férrico), taninos (cloruro férrico, gelatina), alcaloides (Dragendorff, Mayer y Wagner), triterpenos y esteroides (Liebermann-Buchard), quinonas (Bornträger), compuestos lactónicos y cumarinas (Baljet), azúcares reductores (Fehling), antocianidinas (Rosemhein) y saponinas (ensayo de espuma); calificándose como ausencia y presencia si hay cambio de coloración o precipitación ${ }^{(15)}$.

\section{Cuantificación de antocianinas totales}

Se realizó por el método de $\mathrm{pH}$ diferencial reportado por Giusti y Wrolstad (16). Se utilizaron dos sistemas tampón: ácido clorhídrico / cloruro de potasio de $\mathrm{pH}$ 1,0 (o,025 M) y ácido acético / acetato sódico de pH 4,5 (o,4 M). A o,2 mL de la muestra diluida (para conseguir una absorbancia en el rango de 0,100 - 1,200 a $510 \mathrm{\eta m}$ ), se añadieron $1,8 \mathrm{~mL}$ de la correspondiente disolución tampón y se midió la absorbancia frente a un blanco a 510 y $700 \mathrm{\eta m}$. Se calculó la absorbancia final a partir de:

$$
\mathrm{A}=\left(\mathrm{A}_{\max \text { vis }}-\mathrm{A}_{700 \mathrm{~nm}}\right) \mathrm{pH}_{1,0}-\left(\mathrm{A}_{\text {max vis }}-\mathrm{A}_{700 \mathrm{~nm}}\right) \mathrm{pH}_{4,5}
$$

La concentración de pigmentos monoméricos en el extracto se expresó en cianidina-3-glucósido.

$$
\underset{(\mathrm{mg} / 100 \mathrm{~g})}{\operatorname{Antocianinas}} \underset{\mathrm{monoméricas}}{\mathrm{m}}=\frac{\mathrm{A} \text { x PM x FD x } 1000}{(\varepsilon \times 1)}
$$

A = Absorbancia;

$\mathrm{PM}=$ Peso molecular gmol $^{-1}$;

$\mathrm{FD}=$ Factor de dilución;

$\varepsilon=$ Coeficiente de extinción molar, $\mathrm{L} \times \mathrm{mol}^{-1} \times \mathrm{cm}^{-1}$;

$\mathrm{I}=$ Longitud de la trayectoria $(1 \mathrm{~cm})$.

La concentración final de antocianinas $(\mathrm{mg} / 100 \mathrm{~g})$ se calculó en base al volumen de extracto y peso de muestra. Se expresó en cianidina-3-glucósido (PM: 449,2 y $\varepsilon$ : 26 900).

\section{Determinación de polifenoles totales}

Los polifenoles totales en extractos purificados de mashua, se determinaron con el reactivo de FolinCiocalteu, por el método de Singleton y Rossi (17), utilizando ácido gálico como estándar. Se midió la absorbancia a $765 \mathrm{\eta m}$ y los resultados se expresaron como mg equivalentes de ácido gálico (EAG) / 100 g PF.

\section{Evaluación de la actividad antioxidante}

\section{Método DPPH (2,2-difenil-1-picrilhidracilo)}

Se aplicó el método desarrollado por Brand-Willams ${ }^{(18)}$, que se basa en la reducción de la absorbancia medida a 515 ๆm del radical DPPH •, por antioxidantes; que consistió en la medida de la absorbancia del radical DPPH 100 $\mu \mathrm{M}(3,9 \mathrm{~mL})$ disuelto en metanol al 8o \%. Se añadió o,1 $\mathrm{mL}$ de la muestra o estándar, la mezcla se homogenizó cuidadosamente, y se mantuvo en la oscuridad durante 30 minutos. Las medidas de absorbancia a $515 \eta \mathrm{m}$ se realizaron antes de añadir la muestra $\left(A_{0}\right)$ y pasados los 30 y 60 minutos $\left(A_{f}\right)$. La concentración de DPPH• en el medio de reacción se calculó a partir de una curva de calibración obtenida por regresión lineal. Los resultados se expresaron en actividad antioxidante equivalente a Trolox (TEAC). El antioxidante sintético de referencia Trolox, a una concentración de 0,08 - 1,28 mM en disolución de metanol al 80\%, se ensayó en las mismas condiciones.

\section{MétodoABTS(Ácido2,2'-Azinobis-(3-etilbenzotiazolina) -6-sulfónico)}

Se aplicó el método descrito por Kuskoski et al (19), el radical $\mathrm{ABTS}^{\bullet+}$ se obtiene tras la reacción de ABTS (7 $\mathrm{mM})$ con persulfato potásico $(2,45 \mathrm{mM}$, concentración final) incubados a temperatura ambiente $\left( \pm 25^{\circ} \mathrm{C}\right)$ y en la oscuridad durante $16 \mathrm{~h}$. Una vez formado el radical ABTS $^{*+}$ se diluye con etanol hasta obtener un valor de absorbancia comprendido entre 0,70 $( \pm 0,1)$ a $754 \eta \mathrm{m}$. Las muestras filtradas se diluyeron con etanol hasta que se produjo inhibición de 20 a $80 \%$, en comparación con la absorbancia del blanco, tras añadir $20 \mu \mathrm{L}$ de la muestra. A $980 \mu \mathrm{L}$ de dilución del radical $\mathrm{ABTS}^{\bullet+}$, así generado, se le determinó la $\mathrm{A}_{754}$ a $30^{\circ} \mathrm{C}$, se añadió $20 \mu \mathrm{L}$ de la muestra y se midió de nuevo la $\mathrm{A}_{754}$ pasado 1 minuto. La absorbancia se midió de forma continua transcurridos 7 minutos. El antioxidante sintético de referencia, Trolox, se ensayó a una concentración de o - $15 \mu \mathrm{M}$ en etanol, en las mismas condiciones, lo que se hace también con ácido ascórbico (o - $20 \mathrm{mg} / 100 \mathrm{~mL}$ ). Los resultados se expresaron en TEAC y en VCEAC (actividad antioxidante equivalente a vitamina $\mathrm{C}$ ).

\section{Determinación de la concentración de antocianinas empleadas para colorear el yogur}

Se utilizó los parámetros del sistema CIEL*a*b*, reportado por Wrolstad et al ${ }^{(20)}$; se midió el color de 3 marcas de yogur de mora comercial (A, B y C) utilizando los parámetros L* (luminosidad), a* (rojo) y b* (amarillo), los valores de $\mathrm{C}^{*}$ (croma) y h (ángulo de tono) fueron calculados utilizando las ecuaciones (3) y (4).

$$
\begin{aligned}
& \mathrm{C}^{*}=\left[\left(\mathrm{a}^{*}\right) 2+\left(\mathrm{b}^{*}\right) 2\right]^{1 / 2} \\
& \mathrm{~h}=\arctan \left(\mathrm{b}^{*} / \mathrm{a}^{*}\right)
\end{aligned}
$$

Se probaron diferentes concentraciones de los extractos de antocianinas (o,1; 0,2; 0,5; o,8; 1,0; 1,2 y 1,5 $\mathrm{mg} / 100 \mathrm{~g}$ de yogur) y se eligió la que permitía obtener los valores de croma y tono más parecidos a los yogures comerciales de mora, que se ubicaron en el rango de 344,6 - $347,5^{\circ}$ para tono (correspondiendo a un color morado, 
en la escala CIEL*a*b*) y de 51,2 - 53,0 para croma (a valores más altos, colores más vivos). La concentración seleccionada fue 1,2 $\mathrm{mg}$ de antocianinas / $100 \mathrm{~g}$ de yogur. Dada la variabilidad de los parámetros de color entre los yogures, se eligió como referencia el yogur de mora de la marca "B", que para su identificación se le codificó con el número aleatorio " 6224 ", con valores de $344,6^{\circ}$ y 51,2 para tono y croma, respectivamente.

\section{Aplicación y evaluación del pigmento en el yogur natural}

Se evaluó según el método descrito por Salinas et al (21), se definió como unidad experimental $100 \mathrm{~g}$ de yogur natural a los cuales se adicionó 1,2 $\mathrm{mg}$ del extracto etanólico de Tropaeolum tuberosum Ruiz \& Pavón (mashua), codificándose con el número aleatorio " 3500 ". El extracto se incorporó mediante agitación manual hasta su completa disolución. Las variables evaluadas fueron: $\mathrm{pH}$, color, apariencia física (formación de grumos o gránulos de color). Finalmente, se evaluó la estabilidad del pigmento midiendo el color (luminosidad, tono y croma) y el $\mathrm{pH}$. Las mediciones se realizaron el día de preparación de los tratamientos (día cero) y posteriormente cada 4 días a lo largo de 28 días, periodo aproximado que dura la vida de anaquel del yogur. Las muestras se mantuvieron en refrigeración a $4 \pm 1^{\circ} \mathrm{C}$. Los experimentos fueron reproducidos 3 veces.

\section{Evaluación sensorial}

Se aplicó la prueba de aceptación según lo reportado por Anzaldúa-Morales ${ }^{(22)}$, con el objetivo de evaluar la aceptación que podría tener el yogur color morado coloreado con el extracto de antocianinas de Tropaeolum tuberosum Ruiz \& Pavón, analizando la preferencia de los consumidores con respecto a una marca comercial conocida y su actitud hacia el producto y su disposición para comprarlo. Fueron sometidosa evaluación sensorial, con la participación de 80 jueces no entrenados consumidores habituales de yogur. A este grupo de jueces se les aplicó primero un cuestionario para determinar su nivel socioeconómico y cultural. Después se entregó a cada uno un envase con una muestra de cada yogur, marcado con números aleatorios, y el cuestionario para la prueba de aceptación, con el fin de que indiquen su preferencia por alguno de los dos productos. Se determinó, por medio de la tabla de significancia para pruebas de dos muestras $(p<0,05)$, cuál de los dos productos es significativamente más preferido por los jueces.

\section{Análisis estadístico}

Los datos cuantitativos se presentan como valores medios con los respectivos valores de desviación estándar correspondiente a tres repeticiones. La capacidad antioxidante, antocianinas y fenólicos totales se procesaron mediante el análisis unidireccional de la varianza (ANOVA); las diferencias significativas entre medias se determinaron utilizando la prueba de Duncan $(p<0,05)$. Se utilizó el programa SPSS versión 22.0 para las pruebas estadísticas.

\section{RESULTADOS}

Los resultados se muestran en las tablas $1-4$ y en las figuras 2-4.

Tabla 1. Identificación de grupos fitoquímicos (metabolitos secundarios) en el extracto etanólico de Tropaeolum tuberosum Ruiz \& Pavón (mashua).

\begin{tabular}{|c|c|c|c|}
\hline $\begin{array}{l}\text { Metabolitos } \\
\text { secundarios } \\
\end{array}$ & Resultado & Reactivo & Reacción positiva \\
\hline Flavonoides & +++ & Shinoda & Coloración rojiza \\
\hline Fenoles & +++ & $\begin{array}{l}\text { Cloruro } \\
\text { férrico }\end{array}$ & Coloración azul/verde \\
\hline Taninos & ++ & Gelatina & Precipitado blanco \\
\hline Antocianidinas & ++ & Rosemhein & $\begin{array}{l}\text { Coloración en tonos } \\
\text { rojo violeta }\end{array}$ \\
\hline Quinonas & + & Bornträger & $\begin{array}{l}\text { Coloración púrpura o } \\
\text { violeta }\end{array}$ \\
\hline Saponinas & + & $\begin{array}{l}\text { Ensayo de } \\
\text { espuma }\end{array}$ & $\begin{array}{l}\text { Formación de espuma, } \\
\text { y su permanencia por } \\
\text { dos minutos }\end{array}$ \\
\hline Lactonas & - & Bajlet & Coloración violeta \\
\hline Cardenólidos & - & Kedde & $\begin{array}{l}\text { Formación de un anillo } \\
\text { en la interfase color rojo }\end{array}$ \\
\hline $\begin{array}{l}\text { Triterpenos y/o } \\
\text { esteroides }\end{array}$ & $\begin{array}{l}+ \\
+\end{array}$ & $\begin{array}{l}\text { Lieberman- } \\
\text { Burchard } \\
\text { Mayer }\end{array}$ & $\begin{array}{l}\text { Coloración rojo violeta } \\
\text { Precipitado blanco }\end{array}$ \\
\hline Alcaloides & $\begin{array}{l}+ \\
+\end{array}$ & $\begin{array}{l}\text { Dragendorff } \\
\text { Wagner }\end{array}$ & $\begin{array}{l}\text { Precipitado rojo o naranja } \\
\text { Precipitado marrón }\end{array}$ \\
\hline Azucares reductores & + & Fehling & Precipitado rojo ladrillo \\
\hline
\end{tabular}

(-): Ausencia; (+): Trazas; (++): Cantidad moderada; (+++): Cantidad abundante

Tabla 2. Antocianinas y polifenoles totales en el extracto de Tropaeolum tuberosum Ruiz \& Pavón (mashua).

\begin{tabular}{lccc}
\hline \multicolumn{1}{c}{ Características } & Estándar & $\%$ CV & $\begin{array}{c}\text { Contenido } \\
\text { (mg equivalente/100 g PF)* }\end{array}$ \\
\hline Antocianinas & Cianidina-3-glucósido & 9,75 & $192,63 \pm 1,28$ \\
Polifenoles totales $^{(1)}$ & Ácido gálico & 1,46 & $314,12 \pm 1,23$ \\
\hline
\end{tabular}

* Datos expresados en media $\pm \mathrm{DE} ; \quad \mathrm{n}=3$;

$\mathrm{CV}=$ Coeficiente de variabilidad entre repeticiones

(1) $Y=0,1158 X-0,0179 ; R^{2}=0,9986$

Tabla 3. Determinación de la actividad antioxidante del extracto de Tropaeolum tuberosum Ruiz \& Pavón (mashua) aplicando métodos DPPH y ABTS.

\begin{tabular}{ccccccc}
\hline \multirow{3}{*}{ Muestra } & \multicolumn{4}{c}{ TEAC $^{\mathrm{a}}$} & \multicolumn{2}{c}{ VCEAC $^{\mathrm{b}}$} \\
\cline { 2 - 7 } & \multicolumn{2}{c}{ DPPH } & ABTS & DPPH & ABTS \\
\cline { 2 - 7 } & $30 \mathrm{~min}$ & $60 \mathrm{~min}$ & $1 \mathrm{~min}$ & $7 \mathrm{~min}$ & $30 \mathrm{~min}$ & $1 \mathrm{~min}$ \\
\hline $\begin{array}{c}\text { Tropaeolum tuberosum } \\
\text { Ruiz \& Pavón (mashua) }\end{array}$ & $14,2 \pm 0,2$ & $15,8 \pm 0,3$ & $16,3 \pm 0,2$ & $17,0 \pm 0,2$ & $151 \pm 0,3$ & $216 \pm 0,2$ \\
\hline
\end{tabular}

(a) TEAC : Actividad antioxidante equivalente a Trolox ( $\mu \mathrm{mol}$ TE/g peso muestra)

(b) VCEAC: Actividad antioxidante equivalente a ácido ascórbico (vitamina C) ( $\mathrm{mg} / 100 \mathrm{~g}$ peso muestra) Datos expresados en media $\pm D E, n=3$.

Tabla 4. Color de los yogures con y sin adición del extracto de antocianinas de Tropaeolum tuberosum Ruiz \& Pavón, al inicio y final del período de evaluación.

\begin{tabular}{|c|c|c|c|c|c|c|}
\hline \multirow{2}{*}{ Tipo } & \multicolumn{3}{|c|}{ Valores iniciales } & \multicolumn{3}{|c|}{ Valores finales } \\
\hline & L & Hue $^{\circ}$ & Croma & L & Hue $^{\circ}$ & Croma \\
\hline $\begin{array}{l}\text { Yogur “6224" con } \\
\text { colorantes sintéticos }\end{array}$ & 61,6 & 344,6 & 51,2 & 61,6 & 344,6 & 51,2 \\
\hline $\begin{array}{l}\text { Yogur “ } 3500 \text { ” con } \\
\text { extracto de Tropaeolum } \\
\text { tuberosum R. \& P. }\end{array}$ & 68,4 & 336,2 & 47,0 & 59,9 & 341,3 & 46,1 \\
\hline
\end{tabular}

${ }^{*}$ : Los datos representan la media $(n=3) . \quad$ L: Luminosidad. Hue ${ }^{\circ}$ Tono. 


\section{DISCUSIÓN}

El incremento del nivel de vida experimentado por muchos países integrados en la sociedad occidental ha implicado una mayor disponibilidad de alimentos, pero también una mayor exigencia en lo que respecta a la calidad de lo que se consume, así como una especial preocupación por todo lo referente al binomio alimentación-salud. En este sentido, en cuanto puede ser considerada como un bien influido por la dieta, la salud suele ser también base de las innovaciones aparecidas en muchos productos alimenticios actualmente comercializados, que tienen éxito por haber sabido adaptarse a las exigencias de un consumidor preocupado por su salud ${ }^{(23)}$.

Schwartz et al. (24), afirmaron que la demanda de alimentos más saludables y seguros ha fomentado una creciente demanda por colorantes procedentes de fuentes naturales. Los beneficios para la salud asociados a muchos pigmentos naturales los convierten en alternativas atractivas frente a los colorantes sintéticos. Todo esto, combinado con la actividad normativa y la concientización de los consumidores, puede conducir a un mayor interés por el empleo de colorantes procedentes de fuentes naturales.

Según la tabla 2, el contenido de antocianinas en Tropaeolum tuberosum Ruiz \& Pavón está en el orden

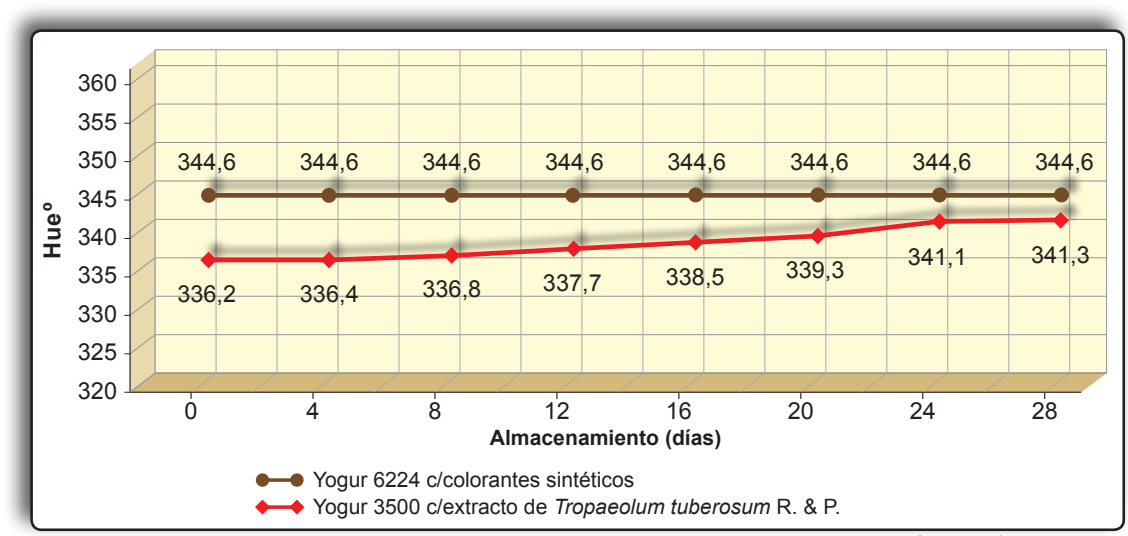

Figura 2. Estabilidad del yogur con y sin adición del extracto de Tropaeolum tuberosum $\mathrm{R}$ \& P., durante 28 días de almacenamiento a $4^{\circ} \mathrm{C}$, valorando Hue ${ }^{\circ}$.

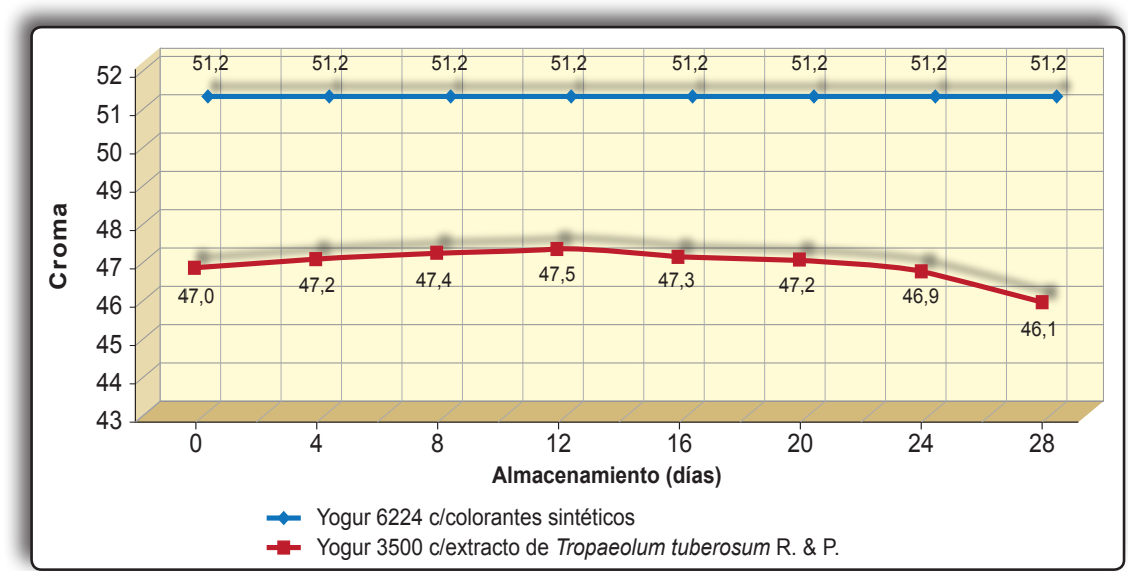

Figura 3. Estabilidad del yogur con y sin adición del extracto de Tropaeolum tuberosum R. \& P., durante 28 días de almacenamiento a $4{ }^{\circ} \mathrm{C}$, valorando croma. de 192,63 \pm 1,28 mg de cianidina-3-glucósido/10o g PF. Por otro lado, se observa que el contenido de polifenoles totales fue de 314,12 $\pm 1,23 \mathrm{mg}$ equivalentes de ácido gálico/10o g PF, con un \% CV de 1,26; lo que indica que no existe variabilidad entre repeticiones. Campos et al (11), reportaron que el contenido total de polifenoles en tubérculos de mashua varió de 0,92 a $3,37 \mathrm{mg}^{-1}{ }^{-1}$, donde los genotipos ARB-5241, DP-02-24 y AGM-5109 tuvieron el mayor contenido total de polifenoles con 3,37; 3,05 y $2,75 \mathrm{mg} \cdot \mathrm{g}^{-1}$, respectivamente, presentando los genotipos de mashua púrpura los valores superiores, mientras que los más bajos correspondieron a los de mashua amarilla. El contenido de antocianinas en genotipos mashua pigmentadas varió de 0,5 a 2,05 mg.g-1; este contenido AT es más alto que aquellos reportados para col roja ( 0,25 mg. $\mathrm{g}^{-1}$ ), fresa $\left(0,15^{-0,3} \mathrm{mg} \cdot \mathrm{g}^{-1}\right)$, frambuesa roja $\left(0,3^{-0}, 4\right.$ mg.g ${ }^{-1}$ y arándano $\left(1,38-3,85 \mathrm{mg}^{-\mathrm{g}^{-1}}\right)$.

Los resultados obtenidos en la determinación de la actividad antioxidante por los métodos indicados (tabla 3), fueron, para VCEAC, $216 \pm 0,2$-para el ensayo ABTS- y $151 \pm 0,3 \mathrm{mg} / 100 \mathrm{~g}$ para el ensayo desarrollado con DPPH. La media de los mayores valores de TEAC fue $17,0 \pm 0,2$ determinado por ABTS y $15,8 \pm 0,1 \mu \mathrm{mol} / \mathrm{g}$ obtenido por DPPH. Se justifica expresarlo en VCEAC debido a que las muestras ensayadas son alimentos, y la vitamina $C$ es un nutriente que se encuentra cotidianamente en nuestra dieta. Dadas las diferencias descritas en los tiempos de medida, en este trabajo se realizó una comparación entre las medias de los valores TEAC obtenidos a los 1 y 7 minutos. Aunque se observa un aumento de los valores TEAC, obtenidos a los 7 minutos, la diferencia entre los resultados obtenidos a 1 minuto no es significativa al nivel de confianza de $95 \%$, por lo que se considera que tiempo de 1 minuto es suficiente para determinar la reactividad de las pulpas de los tubérculos ${ }^{(18)}$.

La intensidad del color morado de los yogures, representado por los valores

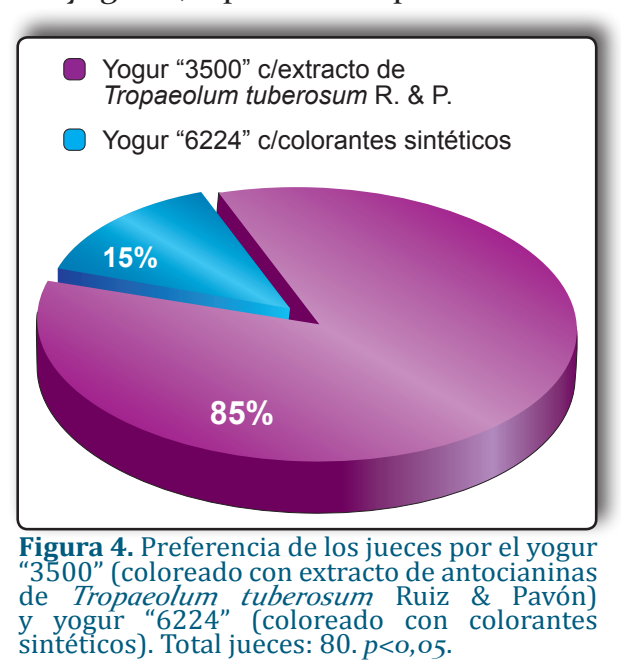


de $C^{*}$, cambiaron ligeramente durante el almacenamiento (figura 3), lo que sugeriría cierta estabilidad del pigmento (14). Esta alteración fue acompañada por cambios de tonalidad, explicados por el leve incremento de los valores de tono o hue de 336,2 a $341,3^{\circ}$ entre los días 8 y 16 , acentuado entre los días 20 y 24 de almacenamiento (figura 2) correspondiente al color púrpura con tonalidad rojiza de acuerdo a la escala CIEL*a*b* (19); los cambios observados en los yogures coloreados se atribuyen a la degradación de las antocianinas del extracto. Salinas et al ${ }^{(21)}$, reportaron similar comportamiento al adicionar a yogur natural los pigmentos de cuatro variedades nativas de maíz (Arrocillo, Peruano, Purepecha y Cónico), asimismo, ocurrió con Aguilera-Ortiz et al ${ }^{(25)}$, al aplicar las antocianinas extraídas de la cáscara de higo (variedad Misión).

En la figura 4 se presentan los resultados de la prueba de aceptación, observándose que el yogur coloreado con el extracto de Tropaeolum tuberosum Ruiz y Pavón es significativamente más preferido por los jueces $(85 \%)$. Se aplicó la prueba de aceptación considerando lo afirmado por Anzaldúa-Morales (22): "el que un alimento le guste a alguien no quiere decir que esa persona vaya a querer comprarlo". El deseo de una persona para adquirir un producto es lo que se llama aceptación, y no sólo depende de la impresión agradable o desagradable que el juez reciba al probar un alimento sino también de aspectos culturales, socioeconómicos, de hábitos, etc.

La calidad de un alimento, sin tomar en cuenta los aspectos sanitarios, toxicológicos y nutricionales, se basa en los siguientes parámetros: color, sabor y olor, y textura. Sin embargo, el primer acercamiento del consumidor al alimento es por su color, ya que relaciona lo adecuado con la aceptación o el rechazo ${ }^{(26)}$. Al ser el color la primera característica del alimento que el consumidor percibe, ejerce gran influencia sobre la elección del alimento, pudiendo determinar la preferencia por un alimento frente a otros de igual o incluso mayor valor nutritivo ${ }^{(2)}$. Schwartz et al (24), afirmaron que el color y el aspecto quizá sean los principales atributos de calidad de los alimentos. Debido a nuestra habilidad para percibir con facilidad estos caracteres, son los primeros en ser evaluados por el consumidor al comprar alimentos.

\section{CONCLUSIONES}

- Los pigmentos extraídos del tubérculo de Tropaeolum tuberosum Ruiz \& Pavón, de piel y pulpa morada, poseen actividad antioxidante y son una alternativa colorante de color morado para su aplicación en alimentos de acidez intermedia como el yogur.

- El tamizaje fitoquímico del extracto contiene abundante cantidad de flavonoides, compuestos fenólicos, antocianidinas y quinonas; que actuando sinérgicamente serían los responsables de la actividad antioxidante.

\section{REFERENCIAS BIBLIOGRÁFICAS}

1. Hashem MM, Atta AH, Arbid MS, Nada SA, Asaad GF. Immunological studies on Amaranth, Sunset Yellow and
Curcumin as food colouring agents in albino rats. Food and Chemical Toxicology. 2010; 48(6): 1581-6.

2. Farré R, Barbera R, Lagarda MJ. Aditivos alimentarios. Capítulo 16. En: Gil A, Editor. Tratado de nutrición. Tomo II. Composición y calidad nutritiva de los alimentos. 2 ed. Madrid: Editorial Médica Panamericana S.A; 2010. p. 427-51.

3. Durante MJ, Pifferi PG, Spagna G, Gilioli E. Partial characterización of Vittis vinifera grape var. ancellota. LWT - Food Science and Technology. 1995; 28(6): 635-7.

4. Waliszewski KN, Blasco G. Propiedades nutracéuticas del licopeno. Salud Pública de México. 2010; 52(3): 254-65.

5. Markakis P. Stability of anthocyanins in foods. En Anthocyanins as Food Colors. P. Markakis (ed.). New York: Academic Press; 1982. p. 1-38.

6. Skrede G, Wrolstad, RE, Lea P, Enersen G. Color stability of strawberry and blackcurrant syrups. Journal of Food Science, 1992; 57(1): 172-7.

7. Rodríguez VM, Simón E. Bases de la alimentación humana. La Coruña: Netbiblo S.L; 2008.

8. Parra RA. Yogur en la salud humana. Revista Lasallista de Investigación. 2012; 9(2): 162-77.

9. Hernández JE, León J. Cultivos marginados: otra perspectiva de 1492. [Internet]. Colección FAO: Producción y protección vegetal; 1992. [Citado 14 mayo 2015]. Disponible en: http://www.fao.org/docrep/o18/ to646s/to646s.pdf

10. Manrique I, Arbizu C, Vivanco F, Gonzales R, Chavez O, Tay D, Ellis D. Tropaeolum tuberosum Ruíz \& Pav. Catálogo de la colección de germoplasma de mashua. Lima: Centro Internacional de la papa; 2014.

11. Campos D, Noratto G, Chirinos R, Arbizu C, Roca W, Cisneros-Zevallos L. Antioxidant capacity and secondary metabolites in four species of Andean tuber crops: native potato (Solanum sp.), mashua (Tropaeolum tuberosum Ruiz \& Pavón), Oca (Oxalis tuberosa Molina) and ulluco (Ullucus tuberosus Caldas). Journal of the Science of Food and Agriculture. 2006; 86(10): 1481-8.

12. Chirinos R, Campos D, Costa N, Arbizu C, Pedreschi $\mathrm{R}$, Larondelle Y. Phenolic profiles of andean mashua (Tropaeolum tuberosum Ruíz \& Pavón) tubers: Identification by HPLC-DAD and evaluation of their antioxidant activity. Food Chemistry. 2008; 106(3): 128598.

13. Yong Ju Z, Howar LR. Effects of solvent and temperature on pressurized liquid extraction of anthocyanins and total phenolics from dried red grape skin. J Agric Food Chem. 2003; (51) 51(18): 5207-13.

14. Vera de Rosso V, Mercadante A. Evaluation of colour and stability of anthocyanins from tropical fruits in an isotonic soft drink system. Innovative Food Science and Emerging Technologies, 2007; 8(3): 347-52.

15. Miranda M, Cuellar A. Manual de prácticas de laboratorio de análisis farmacognóstico. La Habana: Editorial Ciencia y Educación; 1992. p. 23-33.

16. Giusti MM, Wrolstad RE. Unit F1.2.1-13. Anthocyanins. Characterization and measurement with UV-visible spectroscopy. In R. E. Wrolstad (Ed.), Current Protocols in Food Analytical Chemistry. New York: Wiley; 2001. 
17. Singleton VL, Rossi JA. Colorimetry of total phenolics with phosphomolibdic phosphotungstic acid reagent. Am. J. Enol. Vitic, 1965; 16(3): 144-58.

18. Brand-Williams W, Cuvelier ME, Berset C. Use of free radical method to evaluate antioxidant ac $\neg$ tivity. LWT Food Science And Technology. 1995; 28(1): 25-30.

19. Kuskoski EM, Asuero AG, Troncoso AM, Mancini-Filho J, Fett R. Aplicación de diversos métodos químicos para determinar actividad antioxidante en pulpa de frutos. Ciênc Tecnol Aliment. 2005; 25(4): 726-32.

20. Wrolstad RE, Durst RW, Lee J. Tracking color and pigment changes in anthocyanin products. Trends in Food Science \& Technology. 2005; 16(9): 423-8.

21. Salinas Y, Rubio D, Díaz A. Extracción y uso de pigmentos del grano de maíz (Zea mays L.) como colorantes en yogur. ALAN. 2005; 55(3): 293-8.

22. Anzaldúa-Morales A. La evaluación sensorial de los alimentos en la teoría y la práctica. Zaragoza: Editorial Acribia S.A.; 2005.

23. Bello J. Alimentos con beneficios saludables. En: Astiasarán I, Lasheras B, Ariño A, Martínez JA, editores.
Alimentos y nutrición en la práctica sanitaria. Madrid: Ediciones Díaz de Santos S.A.; 2003. p. 35-6o.

24. Schwartz SJ, Von Elbe JH, Giusti MM. Colorantes. En: Damodaran S, Parkin KL, Fennema OR, editores. Fennema Química de los alimentos. 3 ed. Zaragoza: Editorial Acribia S.A; 2010. p. 572-630.

25. Aguilera-Ortíz M, Reza-Vargas MC, Chew Madinaveitia RG, Aguilar-Valenzuela J, Ramírez-Baca P. Antocianinas de higo como colorantes para yogur natural. Revista de Ciencias Biológicas y de la Salud. 2012; XIV(1): 18-24.

26. Guerrero I, López E, Armenta RE. Pigmentos. En: Badui $\mathrm{S}$, editor. Química de los alimentos. México: Pearson Educación de México, S.A. de C.V; 2006. p. 401-44.

Manuscrito recibido el: 24/o6/2015

Aceptado para su publicación el: 02/11/2015

\section{Correspondencia:}

Nombre: Luis Alberto Inostroza Ruiz

Dirección: Jr. Puno 1002- Lima o1-Perú

e-mail: linostrozar@unmsm.edu.pe 